\title{
Mathematical-Physical Properties of Musical Tone Systems III: Tonal Multiplication Tables
}

\author{
By \\ Bruno J. Gruber \\ (Vorgelegt in der Sitzung der math.-nat. Klasse am 17. April 2008 \\ durch das k. M. I. Peter Steinhauser)
}

\begin{abstract}
The familiar tones/intervals of classical western music are analyzed in terms of their "internal" mathematical structure. This leads to mathematical interrelationships in the form of multiplication tables for tones/intervals, factorization of tones and their relationship by means of symmetry operations.
\end{abstract}

\section{Introduction}

It will be shown in this article that the properties of the musical tones and intervals, as encountered in traditional western music, can be described in the form of a multiplication/division table if treated as numerical numbers, and in the form of an addition/subtraction table if considered as vectors. This follows as a consequence from the ("internal") properties of these musical tones and intervals, namely from their property as (scaled) lattice points (vectors). That is, each tone/interval can be factored into a three-fold product of numbers with the three factors given by integer powers of the three prime numbers 2, 3 and 5. This mathematical "inner structure" of the musical tones/intervals permits the introduction of a mathematical (scaled) 3-dimensional lattice system which clearly exhibits 
the properties among the tones/intervals and their interrelationships [1], [2].

Several distinct bases can be used for the mathematical lattice system, all being equivalent. The choice of a particular basis is one of mathematical convenience and/or particular significance to established musical language. MAZZOLA [3] has used a basis for the lattice system which is directly related to the modern tuning methods of musical instruments, while in this article two mathematically convenient bases are being used. One of these two bases is called a "tuning basis", the other is called a "natural basis". Each of these two bases has advantages for different types of calculations as well as for the expression of the properties of the musical tones/ intervals.

It was shown in [2] that the general lattice system of musical tones contains subsystems which form the bases for various musical systems. That is, these musical tonal systems form inventories of tones out of which the tones for actual musical scales can be selected. The musical scales thus form subsets of tones chosen from the musical (inventory) systems ("structured Tonmaterial"). One such system of tones/intervals is given by 116 tones/intervals contained in the octave $\left[c^{0}=c=1 / 1, c^{1}=2 / 1\right]$. These 116 tones/intervals correspond essentially to the tones classified by RIEMANN in [4] in the list "Determination of Tones". Table 10.1 of [2] for the tones/ intervals of the major whole tone $\mathrm{T}_{1}$, the interval $[c=1 / 1, d=9 / 8]$, illustrates this fact, as well as clearly shows the equivalence of the two distinct bases used.

From among the 31 tones/intervals listed in Table 10.1 of [2] for the interval $[c, d], 15$ tones have been selected for the purpose of creating a $15 \times 15$ multiplication/division table (the remaining tones are not relevant for the 116-tone system but belong to other tonal systems). The multiplication/division table for the 15 tones of the interval $[c, d]$, having a total of 225 entries, can be re-expressed, by using symmetry arguments, in the form of a reduced multiplication table containing 120 entries. Of these 120 entries, that is tones/ intervals, only 55 are distinct tones/intervals. Thus, the 15 input tones/intervals are sufficient to generate 40 additional tones/intervals via the multiplication/division table, all of which represent a system of interrelated and interconnected algebraic tones/intervals. The reduced multiplication/division table thus permits in an easy manner to recognize the (algebraic) relationships between tones and intervals, and the factorization of tones and intervals into products of tones and intervals, Sect. 4. 
Having introduced the reduced multiplication table for the interval $[c, d]$ it will be shown that by using the "internal" mathematical structure of the tones/intervals the multiplication table can be extended to the entire octave $\left[c, c^{1}\right]$. Again, using symmetry arguments, the multiplication table for the interval $[c, d]$ can be translated, as a unit, to any other region of the 116-musical-tone system by means of multiplicative factors, or equivalently, by means of adding constant vectors to the entities of the $[c, d]$ multiplication table. This holds for any combination of subintervals of the octave $\left[c, c^{1}\right]$, Sect. 5 .

In Sect. 2 definitions are given and the notation used in this article is described. Moreover, some of the results obtained in [1] and [2] are listed. In Sect. 3 the properties of the natural basis are discussed in some detail. In Sect. 4 a simple example is given for a multiplication table, namely a reduced $7 \times 7$ multiplication/division (addition/subtraction) table, given in terms of well-known tones/ intervals. Sect. 4 thus can serve as an easily understandable introduction to musical multiplication systems in terms of familiar musical terminology. In Sect. 5 the above-mentioned reduced $15 \times 15$ multiplication/subtraction table is discussed, together with a comprehensive list of the 55 musical tones/intervals contained in this table. This list can also serve as a cross reference for the various systems of mathematical and musical characterization of musical tones and intervals. In Sect. 6 the results obtained in Sect. 5 for the interval $[c, d]$ are extended to the octave $\left[c, c^{1}\right]$.

The names used for the musical tones/intervals are those which were defined by HUYGENS-FOKKER [5] (with the exception of a few non-conventional symbols which were introduced for the purpose of suggestive illustration).

\section{Definitions and Notation}

In this section the definitions and the notation used in this article are given.

The tones/intervals $\mathbf{t}$, frequency ratios of musical tone frequencies $\nu_{1} / \nu_{2}$, are characterized in several distinct ways:

a) In the traditional way by means of numbers $\mathbf{t}$ on the real line given by ratios of integers (a one-dimensional approach), $\nu / \nu_{0}$, with the reference frequency $\nu_{0}=c$.

b) By 3-dimensional vectors $\mathbf{t}$ as lattice points in a 3-dimensional mathematical space, with the three dimensions representing intervals 
given by the ratios $2 / 1,3 / 2$, and $5 / 3$,

$$
\begin{aligned}
\mathbf{t} & =\left(k_{1}, k_{2}, k_{3}\right)=(2 / 1)^{k_{1}}(3 / 2)^{k_{2}}(5 / 3)^{k_{3}}, \quad k_{i} \text { integers, } \\
c^{1} / c & =2 / 1, \quad g / c=3 / 2, \quad a / c=5 / 3, \quad\left(c^{0}=c=1\right) .
\end{aligned}
$$

Thus a tone $\mathbf{t}$ can be looked upon simultaneously as representing a vector and a number.

A convenient basis for this tonal system can be defined by

$$
\begin{aligned}
& (1,0,0)=2 / 1=c^{1}, \\
& (0,1,0)=3 / 2=g, \\
& (0,0,1)=5 / 3=a .
\end{aligned}
$$

This basis for tonal systems will be referred to as a "tuning basis". It holds for two tones/intervals

$$
\begin{aligned}
\mathbf{t}_{1} \cdot \mathbf{t}_{2} & =\left(k_{1}, k_{2}, k_{3}\right) \cdot\left(k_{1}^{\prime}, k_{2}^{\prime}, k_{3}^{\prime}\right) \\
& =(2 / 1)^{k_{1}}(3 / 2)^{k_{2}}(5 / 3)^{k_{3}} \cdot(2 / 1)^{k_{1}^{\prime}}(3 / 2)^{k_{2}^{\prime}}(5 / 3)^{k_{3}^{\prime}} \\
& =(2 / 1)^{k_{1}+k_{1}^{\prime}}(3 / 2)^{k_{2}+k_{2}^{\prime}}(5 / 3)^{k_{3}+k_{3}^{\prime}}, \quad \text { as numbers, } \\
& =\left(k_{1}, k_{2}, k_{3}\right)+\left(k_{1}^{\prime}, k_{2}^{\prime}, k_{3}^{\prime}\right)=\left(k_{1}+k_{1}^{\prime}, k_{2}+k_{2}^{\prime}, k_{3}+k_{3}^{\prime}\right) \\
& =\mathbf{t}_{1}+\mathbf{t}_{2}, \quad \text { as vectors. }
\end{aligned}
$$

Thus, in what follows, it will be the composition law, multiplication . or addition + , which will indicate whether a tone is considered to be represented as a number or by the vector corresponding to this number. Moreover, the dot for the multiplication law will be omitted unless it is necessary for a better understanding. Thus we have the equivalence $\mathbf{t}_{1} \cdot \mathbf{t}_{2}=\mathbf{t}_{1}+\mathbf{t}_{2}$.

c) As vectors $\mathbf{t}$ expressed with respect to the basis $\boldsymbol{\lambda}, \boldsymbol{\mu}, \boldsymbol{\rho}$,

$$
\begin{array}{rlr}
\mathbf{t}=[n, m, r]=\lambda^{n} \mu^{m} \rho^{r}, & n, m, r, \quad \text { integers, } \\
\lambda=[1,0,0]=(-6,9,1), & \text { schisma, } \\
\mu=[0,1,0]=(11,-15,-3), & \text { diaschisma-schisma, } \\
\rho=[0,0,1]=(-6,4,5], & \text { small diesis, }
\end{array}
$$

where the names for the base vectors have been taken from ref. [5]. 
This basis will be referred to as the "natural basis". The mathematical form of the basis elements $\lambda, \mu, \rho$ has been obtained in [2] as a consequence of the "closure condition" for a (scaled) tonal system.

The relationship between the musical lattice points, expressed in terms of the tuning basis and the natural basis, is given by

$$
\begin{gathered}
{[n, m, r]=(-6 n+11 m-6 r, 9 n-15 m+4 r, n-3 m+5 r),} \\
(n, m, r)=\left[63 k_{1}+37 k_{2}+46 k_{3}, 41 k_{1}+24 k_{2}+30 k_{3}, 12 k_{1}+7 k_{2}+9 k_{3}\right], \\
n, m, r, k_{1}, k_{2}, k_{3}, \text { integers. }
\end{gathered}
$$

It holds

$2 / 1=c^{1}=(1,0,0)=63[1,0,0]+41[0,1,0]+12[0,0,1]=[63,41,12]$,

$3 / 2=g=(0,1,0)=[37,24,7]$,

$5 / 3=a=(0,0,1)=[46,30,9]$.

The number $N$ of intervals contained in the octave $\left[c, c^{1}\right]$ is given by (see [2]),

$$
N=63+41+12=116 .
$$

d) By an ideographic notation, in the form of symbols like

$\sharp$, for the minor chroma,

$b_{1}$, for the large limma,

$b_{2}$, for the minor diatonic semitone,

etc.

These various types of notation will be used interchangeably as each notation has its own aspect of usefulness.

The 116-tone musical lattice system discussed in [2] contains various musical tonal subsystems. While these musical subsystems are 3-dimensional, the general mathematical lattice system contains also 2-dimensional tonal systems (for example, the Pythagorean-type musical tonal systems). In fact, the system of octave tones itself can be considered as a 1-dimensional musical (sub)system.

The bases for some of the tonal subsystems which were discussed in [2] are listed in what follows:

\subsection{Three-Dimensional Musical Systems}

1) A 116-tone musical lattice system (related to RIEMANN's list of tones, [4]). The bases and definitions for this musical lattice 
system have been given in the preceding section of this article. The tones in list 10.1 of [2] are numbered by $\# n, n=1,2,3, \ldots, 31$. The numbering of the tones discussed in this article is taken from this list.

2) A 31-tone musical lattice subsystem of the 116-tone musical system. The basis for the 31 -tone subsystem is given by

$$
\begin{array}{ll}
\left\{S_{1}^{-1}, S_{1}^{2} S_{2}, S_{1}^{-1} \mathbf{p}^{-1}\right\}, & \\
S_{1}^{-1}=(3,0,-4)=[5,3,0], & \text { major diesis, } \\
S_{2}=(1,1,-2)=[8,5,1], & \text { large limma, } \\
\mathbf{p}=(-1,3,-1)=[2,1,0], & \text { syntonic comma, } \\
& \text { Pythagorean vector, } \\
S_{1}^{2} S_{2}=(-5,1,6)=[-2,-1,1], & \text { Kleisma, } \\
S_{1}^{-1} \mathbf{p}^{-1}=(4,-3,-3)=[3,2,0], & \text { minor diesis, } \\
S_{1} S_{2}=S_{3}=(-2,1,2) & \\
\quad=[3,2,1]=\gamma=\sharp=\text { cis, } & \text { minor chroma, } \\
S_{3}=S_{2} S_{1}^{-1}=(-2,1,2) \cdot(3,0,-4) & \\
=(1,1,-2)=[3,2,1] \cdot[5,3,0] & \\
=[8,5,1]=b_{1}=\text { des, } & \text { large limma, }
\end{array}
$$

with

$\left(S_{1}^{-1}\right)^{15}\left(S_{1}^{2} S_{2}\right)^{12}\left(S_{1}^{-1} \mathbf{p}^{-1}\right)^{4}=2 / 1, \quad$ the octave condition, and $N=15+12+4$, number of tones/intervals.

3) A 23-tone musical lattice subsystem is generated by the intervals $\left\{\alpha, \gamma, \mathbf{p}, \gamma \mathbf{p}^{-1}\right\}$

$$
\begin{array}{ll}
\alpha=(3,-5,0)=[4,3,1]=s_{3}=S p^{-1}, & \text { limma, } \\
\gamma=(-2,1,2)=[3,2,1]=S_{3}=\text { cis, } & \text { minor chroma, } \\
\mathbf{p}=(-1,3,-1)=[2,1,0], & \text { syntonic comma, } \\
& \text { Pythagorean vector, } \\
\gamma \mathbf{p}^{-1}=(-1,-2,3)=[1,1,1], & \text { maximal diesis, } \\
S=(2,-2,-1)=[6,4,1] & \\
=\alpha \mathbf{p}=S_{2} \mathbf{p}^{-1}=b_{2}, & \text { semitone, }
\end{array}
$$




$$
\begin{array}{ll}
\alpha^{7} \mathbf{p}^{11} \gamma^{4}\left(\gamma \mathbf{p}^{-1}\right)=2, & \text { the octave condition, and } \\
N=7+11+4+1, & \text { the number of tones/intervals. }
\end{array}
$$

\subsection{Relationships Between Tonal Systems (for Selected Tones)}

$$
\begin{aligned}
S_{3} & =S_{1} S_{2}=\sharp(\text { sharp })=\mathrm{cis}, \\
s_{3} & =s_{1} s_{2}=S \mathbf{p}^{-1}=\alpha, \\
S & =\alpha \mathbf{p}=b_{2}=S_{2} \mathbf{p}^{-1}, \\
S_{2} & =b_{1}=\mathrm{des}, \\
T_{2} & =T_{1} p^{-1}=\alpha \mathbf{p} \gamma, \\
T_{1} & =S_{1} S_{2}^{2}=d, \\
\rho & =S_{1}^{2} S_{2} \mathbf{p}, \\
\mathbf{p} \gamma \mathbf{p} & =s_{1}^{2} s_{2} .
\end{aligned}
$$

For additional details, and other musical tonal subsystems, see [2].

As it was mentioned it is the operation, addition or multiplication, which defines whether a given symbol is to be used as number or as vector. Thus, in the equations given above, all symbols represent numerical values. Exchanging the multiplication with addtion, all symbols represent 3-dimensional vectors.

\section{Multiplication Table for the Natural Basis $\lambda, \mu$,}

The multiplication table for the three basis elements of the natural basis of the 116-tone musical tone system is given in Table 1. This table is obtained by means of straightforward calculation of the products, or sums, of the basis elements $\boldsymbol{\lambda}, \boldsymbol{\mu}, \boldsymbol{\rho}$. The symbols $\mathbf{f} \rightarrow$ and $\mathbf{f} \downarrow$ denote interval factors. The symbol $\mathbf{f} \rightarrow$ represents the fixed quotient between the tones of two adjacent neighboring columns located along the same horizontal line, while the symbol $\mathbf{f} \downarrow$ represents the fixed quotient between the tones of two adjacent horizontal lines located in the same vertical column. In order to save space a reduced multiplication table is given. Use has been made of symmetry, namely the commutativity of multiplication. The tones $\mathbf{t}_{1}$ are listed horizontally above the double line. The tones $\mathbf{t}_{2}$ are listed diagonally below the diagonal double line. Choosing a tone $\mathbf{t}_{1}$ and a tone $\mathbf{t}_{2}$, the quotient tone $\mathbf{t}_{3}=\mathbf{t}_{1} / \mathbf{t}_{2}$ is found as the entry at the intersection of the vertical line extending from tone $\mathbf{t}_{1}$ and the horizontal line extending from $\mathbf{t}_{2}$. Similarly, multiplication is 
Table 1a. Algebraic Properties of the Natural Basis $\lambda, \mu, \rho$

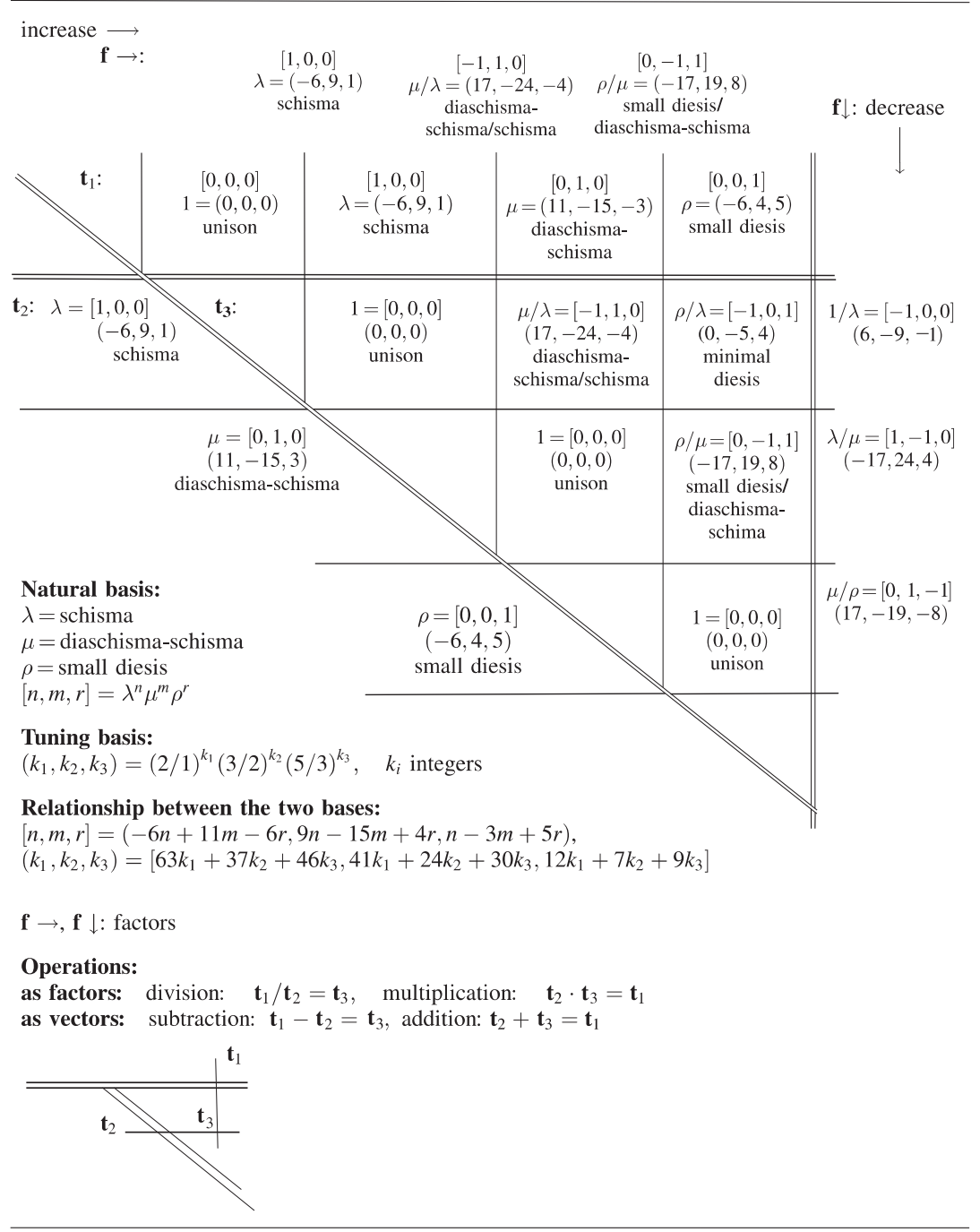

given by choosing a tone $\mathbf{t}_{2}$ and a tone $\mathbf{t}_{3}$, obtaining the product as the tone $\mathbf{t}_{1}=\mathbf{t}_{2} \cdot \mathbf{t}_{3}$ as the tone vertically above the tone $\mathbf{t}_{3}$. As can be easily recognized, the multiplication/division operation can be replaced by vector addition/subtraction.

Thus the internal algebraic structure of the three basis elements $\lambda, \mu, \rho$ gives rise to algebraic relationships among the three basis 
Table 1b. Algebraic Properties of the Natural Basis $\lambda, \mu, \rho$

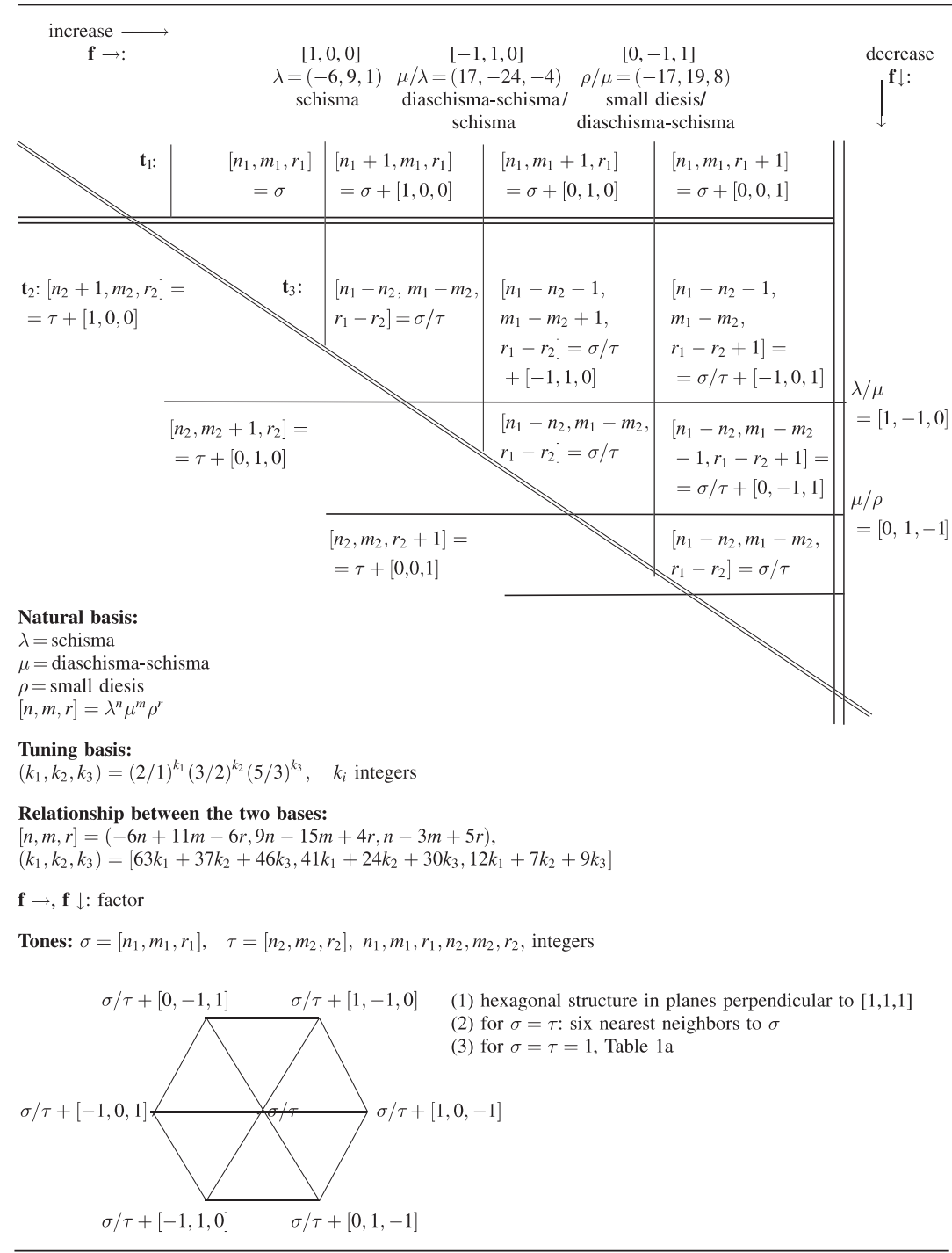

elements. That is, in terms of musical names, the tones

$$
\begin{aligned}
\lambda & =[1,0,0]=(-6,9,1) & & =\text { schisma }, \\
\mu & =[0,1,0]=(11,-15,-3) & & =\text { diaschisma-schisma } \\
\rho & =[0,0,1]=(-6,4,5) & & =\text { small diesis }
\end{aligned}
$$


generate the tones, and relationships between the tones,

$\mu / \lambda=[-1,1,0]=(17,-24,-4)=$ diaschisma-schisma $/$ schisma,

$\rho / \lambda=[-1,0,1]=(0,-5,4) \quad=$ small diesis $/$ schisma

$=$ minimal diesis,

$\rho / \mu=[0,-1,1]=(-17,19,8)=$ small diesis $/$ diaschischimaschisma.

\section{The $7 \times 7$ Tonal Multiplication Table}

The $7 \times 7$ tonal multiplication table provides a simple example, in terms of the familiar musical concepts, for the interrelationships between tones/intervals, and moreover illustrates the algebraic methods discussed in this article.

For the multiplication table 7 tones/intervals are chosen from within the full tone $T_{1}(=[c, d])$, namely

$\mathbf{t}_{1}, \mathbf{t}_{2}$ :

$c / c=1 / 1=1=(0,0,0)=[0,0,0]$, unison,

$\mathbf{p}=81 / 80=(-1,3,-1)=[2,1,0], \quad$ syntonic comma,

Pythagorean vector,

$\sharp=25 / 24=(-2,1,2)=[3,2,1], \quad$ minor chroma,

$b_{2}=16 / 15=(2,-2,-1)=[6,4,1], \quad$ leading tone step,

minor diatonic semitone,

$b_{1}=(1,1,-2)=[8,5,1], \quad$ large limma, BP semitone,

$T_{2}=10 / 9=(0,-1,1)=[9,6,2], \quad$ minor whole tone,

$T_{1}=9 / 8=(-1,2,0)=[11,7,2], \quad$ major whole tone.

The multiplication table then generates additional 6 tones/intervals within the interval $T_{1}$, namely the tones/intervals

$$
\begin{aligned}
& \mathbf{t}_{3}: \\
& \begin{array}{rlr}
\sharp / \mathbf{p} & =250 / 243=(-1,-2,3)=[1,1,1] & \\
& =T_{2} / b_{1}, & \\
b_{2} / \mathbf{p}=256 / 243=(3,-5,0)=[4,3,1]=s_{3}, & & \text { limma, } \\
T_{2} / \mathbf{p}=800 / 729=(1,-4,2)=[7,5,2], & & \text { grave whole tone, }
\end{array}
\end{aligned}
$$




$$
\begin{aligned}
& b_{2} / \sharp=128 / 125=(4,-3,-3)=[3,2,0], \\
& b_{1} / \sharp=648 / 625=(3,0,-4)=[5,3,0], \\
& T_{1} / b_{2}=135 / 128=(-3,4,1)=[5,3,1],
\end{aligned}
$$

minor diesis,

major diesis,

major chroma. (4.2)

Table 2a. $7 \times 7$ Tone Multiplication (Division) Table, Interval $c-d$, Major Whole Tone $T_{1}$

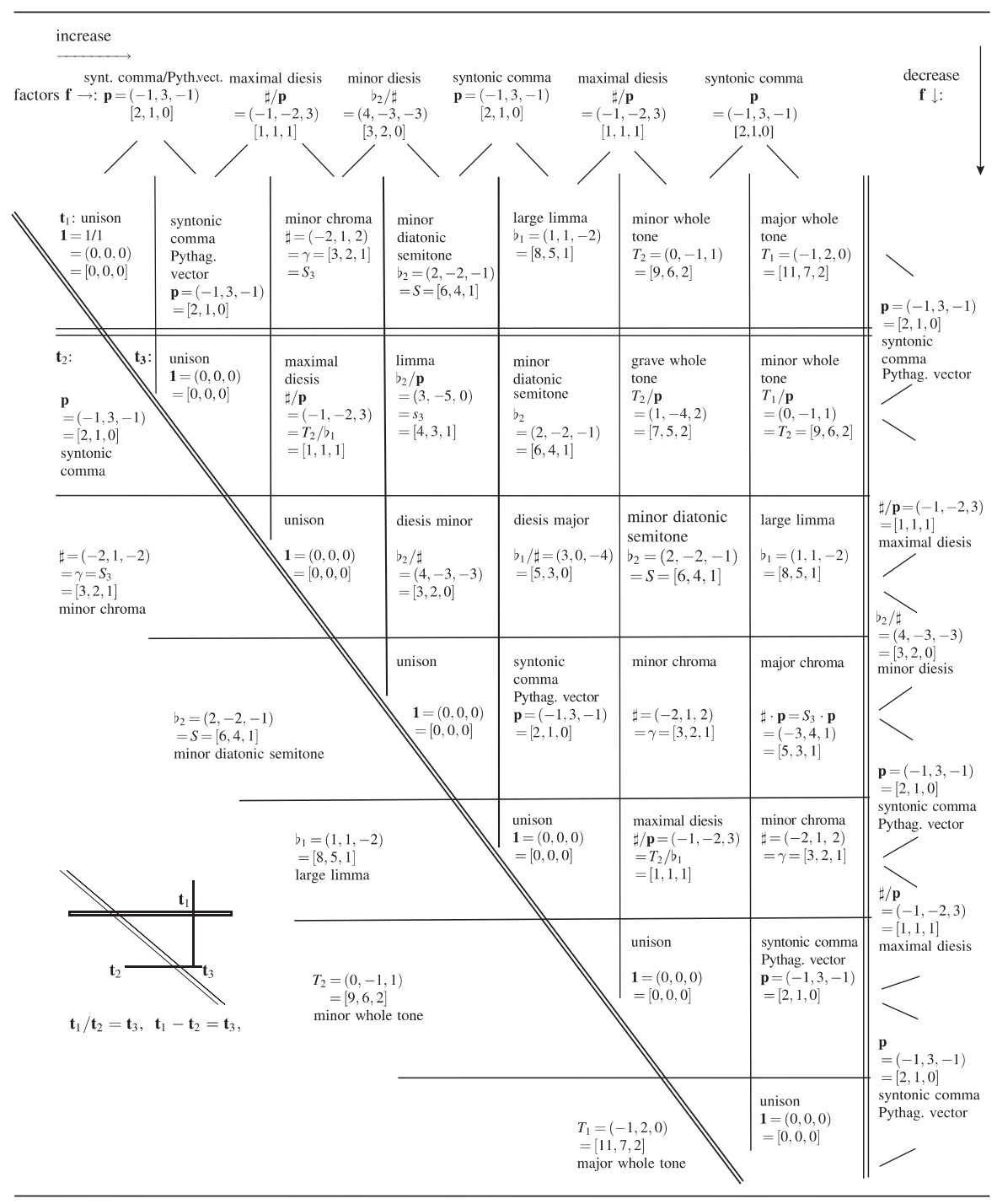


Table $2 b$

Multiplication Table 2a serves as an easily recognizable and understandable example for the general multiplication tables for tones (intervals) of the octave $c-c^{1}$. The table permits multiplication and division of tones (and equivalently addition and subtraction of the tonal vectors). For division (subtraction) holds $\mathbf{t}_{1} / \mathbf{t}_{2}=\mathbf{t}_{3}$, with $\mathbf{t}_{3}$ given by the tone at the intersection of the vertical line from the tone $\mathbf{t}_{1}$ with the horizontal line from the tone $\mathbf{t}_{2}$. Similarly, for multiplication (addition) holds $\mathbf{t}_{2} \cdot \mathbf{t}_{3}=\mathbf{t}_{1}$, with $\mathbf{t}_{2}$ and $\mathbf{t}_{3}$ along a horizontal line and $\mathbf{t}_{1}$ given by the vertical line up. The factors $\mathbf{f}$ (vectors $\mathbf{f}$ ) connecting neighboring tones are given on top, and to the right, of the multiplication table.

Table $2 b$ lists the tones (intervals) which occur as entries in the $7 \times 7$ multiplication table shown in Table 2a. The 7 input tones represent 7 (familiar) tones selected, for the purpose of demonstration, from among the tones of the basic octave $c-c^{1}$. The resultant multiplication (division) table thus has 49 tones as entries. Symmetry considerations lead to a reduced multiplication table containing 28 tones as entries. Of these 28 tones 13 tones represent distinct tones, listed below. Of the 13 distinct tones 7 are the (arbitrarily selected) input tones, while 6 tones are generated by means of the multiplication table as a consequence of the (mathematical) properties of the musical tones. The generated tones represent the interrelationships which exist between the 13 tones of Table $2 \mathrm{a}$.

For purposes of demonstration of the multiplication table, and moreover in order to clearly demonstrate the existing correlations between the various notations, the tones have been characterized by means of various types of notation, ranging from mathematical descriptions to ideographic description and description by means of tone names. Each of these notations has its advantages depending upon the type of calculation which is to be performed.

To summarize:

\section{Input tones:}

$$
\begin{aligned}
& \mathbf{1}=(0,0,0)=[0,0,0]=1 / 1, \quad \text { unison, } \\
& \mathbf{p}=(-1,3,-1)=[2,1,0]=81 / 80, \quad \text { syntonic comma, Didymic comma, } \\
& \text { Pythagorean vector, } \\
& \sharp=(-2,1,2)=[3,2,1]=25 / 24, \quad \text { minor chroma, cis, } \gamma, S_{3} \text {, } \\
& b_{2}=(2,-2,-1)=[6,4,1]=16 / 15, \quad \text { minor diatonic semitone, } S \text {, } \\
& b_{1}=(1,1,-2)=[8,5,1]=27 / 25, \quad \text { large limma, des, } \\
& T_{2}=(0,-1,1)=[9,6,2]=10 / 9, \quad \text { minor whole tone, } \\
& T_{1}=(-1,2,0)=[11,7,2]=9 / 8, \quad \text { major whole tone. }
\end{aligned}
$$

\section{Generated tones:}

$$
\begin{aligned}
\sharp / \mathbf{p} & =(-1,-2,3)=[1,1,1]=250 / 243=T_{2} / b_{1}, & & \text { maximal diesis, } \\
b_{2} / \mathbf{p} & =(3,-5,0)=[4,3,1]=256 / 243=s_{3}, & & \text { limma, } \\
T_{2} / \mathbf{p} & =(1,-4,2)=[7,5,2]=800 / 729, & & \text { grave whole tone, } \\
b_{\mathbf{2}} / \sharp & =(4,-3,-3)=[3,2,0]=128 / 125, & & \text { minor diesis, }
\end{aligned}
$$


Table 2b (continued)

$$
\begin{aligned}
b_{1} / \sharp & =(3,0,-4)=[5,3,0]=648 / 625, & & \text { major diesis, } \\
T_{1} / b_{2} & =(-3,4,1)=[5,3,1]=135 / 128, & & \text { major chroma. }
\end{aligned}
$$

Def: "tuning basis"

$$
\begin{aligned}
\left(k_{1}, k_{2}, k_{3}\right) & =(2 / 1)^{k_{1}}(3 / 2)^{k_{2}}(5 / 3)^{k_{3}}, \quad k_{i} \text { integers } \\
(1,0,0) & =2 / 1=\text { tone } c^{1} \\
(0,1,0) & =3 / 2=\text { tone } g \\
(0,0,1) & =5 / 3=\text { tone } a .
\end{aligned}
$$

Def: "natural basis"

$$
\begin{aligned}
{[n, m, r] } & =\lambda^{n} \mu^{m} \rho^{r}, & & n, m, r, \text { integers }, \\
\lambda & =(-6,9,1), & & \text { schisma, } \\
\mu & =(11,-15,-3), & & \text { diaschisma-schisma, } \\
\rho & =(-6,4,5), & & \text { small diesis. }
\end{aligned}
$$

Relationship between the two bases:

$$
\begin{aligned}
{[n, m, r] } & =(-6 n+11 m-6 r, 9 n-15 m+4 r, n-3 m+5 r), \\
\left(k_{1}, k_{1}, k_{3}\right) & =\left[63 k_{1}+37 k_{2}+46 k_{3}, 41 k_{1}+24 k_{2}+30 k_{3}, 12 k_{1}+7 k_{2}+9 k_{3}\right] .
\end{aligned}
$$

\section{The $15 \times 15$ Multiplication Table for the Interval $[c, d]$}

In this section the multiplication table for 15 subintervals of the major whole tone $T_{1}$, the interval $[c, d]$, is discussed. The 15 subintervals have been chosen from Table 10.1 of [2]. The number \# $n$ of a tone in the multiplication table refers to the number of this tone given in Table 10.1. The particular choice of subintervals of $[c, d]$ is motivated by the fact that these subintervals belong to the 116-tone musical system (the other musical subsystems being ignored in this context).

The choice of 15 input tones leads to a multiplication table containing 225 tonal entries. Using again symmetry arguments, the commutativity of multiplication, a reduced multiplication table containing 120 tonal entries is obtained. Among the 120 entries are 55 distinct tones/intervals, listed in Table 4. Thus, the algebraic properties of the 15 tones selected generate 40 additional tones within the interval $[c, d]$. The table represents a consistent tonal system, and clearly exhibits properties and relationships among the tones. 
Table 3. $15 \times 15$ Multiplication Table for Musical Tones/Intervals (Addition/Subtraction). The number symbol \# denotes the number of the tone as listed in Table 10.1 of ref. [2]. This list corresponds essentially to the list of tones obtained by RIEMANN, ref. [4]. The symbols $\mathbf{p}, S, S_{1}, S_{2}$, etc., which occur in this table, refer to tonal interval systems which were discussed in ref. [2]

\begin{tabular}{|c|c|c|}
\hline $\begin{array}{l}c^{1}=c=(2 / 1) \\
g=(3 / 2) \\
a=(5 / 3)\end{array}$ & $(n, m, r)=c^{n} g^{m} a^{r}$ & $\begin{array}{l}\lambda=(-6,9,1) \\
\mu=(11,-15,-3) \\
\rho=(-6,4,5)\end{array}$ \\
\hline
\end{tabular}

$\mathbf{t}_{2} \cdot \mathbf{t}_{3}=\mathbf{t}_{1}$ (for details see also previous tables)

\begin{tabular}{|c|c|c|c|c|c|c|c|c|}
\hline $\begin{array}{l}\text { factors: } \\
\mathrm{f} \rightarrow\end{array}$ & $\begin{array}{l}\lambda=(-6,9,1) \\
\text { schisma }\end{array}$ & $\begin{array}{l}\mu / \lambda= \\
(17,-24,-4)\end{array}$ & $\begin{array}{l}\lambda=(-6,9,1) \\
\text { schisma }\end{array}$ & $\begin{array}{l}\lambda=(-6,9,1) \\
\text { schisma }\end{array}$ & $\begin{array}{l}\lambda=(-6,9,1) \\
\text { schisma }\end{array}$ & $\begin{array}{l}\rho / \lambda^{3} \mu= \\
(1,-8,5) \\
\text { Kleisma- } \\
\text { schisma }\end{array}$ & $\begin{array}{l}\lambda^{3} \mu^{2} / \rho= \\
(10,-7,-8) \\
\text { Wuerschmidt's } \\
\text { comma }\end{array}$ & $\begin{array}{l}\rho=(-6,4,5) \\
\text { small diesis }\end{array}$ \\
\hline $\begin{array}{l}\# \text { of } \\
\text { tone }\end{array}$ & $\# 1$ & $\# 2$ & $\# 4$ & $\# 5$ & $\# 6$ & $\# 7$ & $\# 8$ & $\# 9$ \\
\hline tone $t_{1}$ : & $\begin{array}{l}\mathbf{1}=(0,0,0) \\
\text { unison }\end{array}$ & $\begin{array}{l}\lambda=(-6,9,1) \\
\text { schisma }\end{array}$ & $\begin{array}{l}\mu= \\
(11,-15,-3) \\
\text { diaschisma- } \\
\text { schisma }\end{array}$ & $\begin{array}{l}\lambda \mu= \\
(5,-6,-2) \\
\text { diaschisma }\end{array}$ & $\begin{array}{l}\lambda^{2} \mu= \\
(-1,3,-1) \\
\text { syntonic } \\
\text { comma, }=\mathbf{p}\end{array}$ & $\begin{array}{l}\lambda^{3} \mu= \\
(-7,12,0) \\
\text { Pythagorean } \\
\text { comma, }=\mathbf{s}_{1}\end{array}$ & $\begin{array}{l}\rho=(-6,4,5) \\
\text { small diesis }\end{array}$ & $\begin{array}{l}\lambda^{3} \mu^{2}= \\
(4,-3,-3) \\
\text { minor diesis, } \\
=\mathbf{S}_{1}^{-1} \mathbf{p}^{-1}\end{array}$ \\
\hline \multirow[t]{9}{*}{$\mid \begin{array}{l}\text { tone } \mathbf{t}_{\mathbf{2}}: \\
\lambda= \\
(-6,9,1) \\
\text { schisma }\end{array}$} & & $\begin{array}{l}\mathbf{1}=(0,0,0) \\
\text { unison }\end{array}$ & $\begin{array}{l}\mu / \lambda= \\
(17,-24,-4)\end{array}$ & $\begin{array}{l}\mu= \\
(11,-15,-3) \\
\text { diaschisma- } \\
\text { schisma }\end{array}$ & $\begin{array}{l}\lambda \mu= \\
(5,-6,-2) \\
\text { diaschisma }\end{array}$ & $\begin{array}{l}\lambda^{2} \mu= \\
(-1,3,-1) \\
\text { syntonic } \\
\text { comma, }=\mathbf{p}\end{array}$ & $\begin{array}{l}\rho / \lambda=(0,-5,4) \\
\text { minimal } \\
\text { diesis }\end{array}$ & $\begin{array}{l}\lambda^{2} \mu^{2}= \\
(10,-12,-4) \\
\text { (diaschisma) }\end{array}$ \\
\hline & $\begin{array}{l}\mu= \\
(11,-15,-3) \\
\text { diaschisma- } \\
\text { schisma }\end{array}$ & & $\begin{array}{l}\mathbf{1}=(0,0,0) \\
\text { unison }\end{array}$ & $\begin{array}{l}\lambda=(-6,9,1) \\
\text { schisma }\end{array}$ & $\begin{array}{l}\lambda^{2}= \\
(-12,18,2) \\
(\text { schisma })^{2}\end{array}$ & $\begin{array}{l}\lambda^{3}= \\
(-18,27,3) \\
(\text { schisma) })^{3}\end{array}$ & $\begin{array}{l}\rho / \mu= \\
(-17,19,8)\end{array}$ & $\begin{array}{l}\lambda^{3} \mu=(-7,12,0) \\
\text { Pythagorean } \\
\text { comma, }=\mathbf{s}_{1}\end{array}$ \\
\hline & & $\begin{array}{l}\lambda \mu= \\
(5,-6,-2) \\
\text { diaschisma }\end{array}$ & & $\begin{array}{l}\mathbf{1}=(0,0,0) \\
\text { unison }\end{array}$ & $\begin{array}{l}\lambda=(-6,9,1) \\
\text { schisma }\end{array}$ & $\begin{array}{l}\lambda^{2}= \\
(-12,18,2) \\
(\text { schisma })^{2}\end{array}$ & $\begin{array}{l}\rho / \lambda \mu= \\
(-11,10,7) \\
\text { semicomma, } \\
\text { Fokker's comma }\end{array}$ & $\begin{array}{l}\lambda^{2} \mu=(-1,3,-1) \\
\text { syntonic } \\
\text { comma, }=\mathbf{p}\end{array}$ \\
\hline & & & $\begin{array}{l}\lambda^{2} \mu= \\
(-1,3,-1) \\
\text { syntonic } \\
\text { comma, }=\mathbf{p}\end{array}$ & & $\begin{array}{l}\mathbf{1}=(0,0,0) \\
\text { unison }\end{array}$ & $\begin{array}{l}\lambda=(-6,9,1) \\
\text { schisma }\end{array}$ & $\begin{array}{l}\rho / \lambda^{2} \mu= \\
(-5,1,6) \\
\text { Kleisma }\end{array}$ & $\begin{array}{l}\lambda \mu=(5,-6,-2) \\
\text { diaschisma }\end{array}$ \\
\hline & & & & $\begin{array}{l}\lambda^{3} \mu= \\
(-7,12,0) \\
\text { Pythagorean } \\
\text { comma, }=\mathbf{s}_{1}\end{array}$ & & $\begin{array}{l}\mathbf{1}=(0,0,0) \\
\text { unison }\end{array}$ & $\begin{array}{l}\rho / \lambda^{3} \mu= \\
(1,-8,5) \\
\text { Kleisma- } \\
\text { schisma }\end{array}$ & $\begin{array}{l}\mu=(11,-15,-3) \\
\text { diaschisma- } \\
\text { schisma }\end{array}$ \\
\hline & & & & & $\begin{array}{l}\rho=(-6,4,5) \\
\text { small diesis }\end{array}$ & & $\begin{array}{l}\mathbf{1}=(0,0,0) \\
\text { unison }\end{array}$ & $\begin{array}{l}\lambda^{3} \mu^{2} / \rho= \\
(10,-7,-8) \\
\text { Wuerschmidt's } \\
\text { comma }\end{array}$ \\
\hline & & & & & & $\begin{array}{l}\lambda^{3} \mu^{2}= \\
(4,-3,-3) \\
\text { minor diesis, } \\
=\mathbf{S}_{1}{ }^{-1} \mathbf{p}^{-1}\end{array}$ & & $\begin{array}{l}\mathbf{1}=(0,0,0) \\
\text { unison }\end{array}$ \\
\hline & & & & & & & $\begin{array}{l}\lambda^{3} \mu^{2} \rho= \\
(-2,1,2) \\
\text { minor chroma, } \\
=\mathbf{S}_{3}=\gamma\end{array}$ & \\
\hline & & & & & & & & $\begin{array}{l}\lambda^{4} \mu^{3} \rho=(3,-5,0) \\
\text { limma, }=\mathbf{s}_{3}=\boldsymbol{\alpha}\end{array}$ \\
\hline
\end{tabular}


Table 3 (continued)

\begin{tabular}{|c|c|c|c|c|c|c|c|}
\hline $\begin{array}{l}\lambda \mu=(5,-6,-2) \\
\text { diaschisma }\end{array}$ & $\begin{array}{l}\lambda=(-6,9,1) \\
\text { schisma }\end{array}$ & $\begin{array}{l}\lambda \mu=(5,-6,-2) \\
\text { diaschisma }\end{array}$ & $\begin{array}{l}\lambda^{2} \mu=(-1,3,-1) \\
\text { syntonic } \\
\text { comma, }=\mathbf{p}\end{array}$ & $\begin{array}{l}\lambda \mu \rho=(-1,-2,3) \\
\text { maximal } \\
\text { diesis }\end{array}$ & $\begin{array}{l}\lambda^{2} \mu=(-1,3,-1) \\
\text { syntonic } \\
\text { comma, }=\mathbf{p}\end{array}$ & & $\begin{array}{l}\text { factors: } \\
\mathbf{f} \downarrow\end{array}$ \\
\hline$\# 14$ & $\# 16$ & $\# 17$ & $\# 19$ & $\# 23$ & \#28 & $\# 31$ & \\
\hline $\begin{array}{l}\lambda^{3} \mu^{2} \rho= \\
(-2,1,2) \\
\text { minor } \\
\text { chroma, } \\
=\mathbf{S}_{3}=\gamma\end{array}$ & $\begin{array}{l}\lambda^{4} \mu^{3} \rho= \\
(3,-5,0) \\
\text { limma, } \\
=\mathbf{s}_{3}=\boldsymbol{\alpha}\end{array}$ & $\begin{array}{l}\lambda^{5} \mu^{3} \rho= \\
(-3,4,1) \\
\text { major } \\
\text { chroma, } \\
\text { major limma }\end{array}$ & $\begin{array}{l}\lambda^{6} \mu^{4} \rho= \\
(2,-2,-1) \\
\text { minor } \\
\text { diatonic } \\
\text { semitone, }=\mathbf{S}\end{array}$ & \begin{tabular}{|l}
$\lambda^{8} \mu^{5} \rho=$ \\
$(1,1,-2)$ \\
large limma, \\
BP sm. \\
semitone, $\mathbf{S}_{2}$
\end{tabular} & $\begin{array}{l}\lambda^{9} \mu^{6} \rho^{2}= \\
(0,-1,1) \\
\text { minor whole } \\
\text { tone, }=\mathbf{T}_{2}\end{array}$ & $\begin{array}{l}\lambda^{11} \mu^{7} \rho^{2}= \\
(-1,2,0) \\
\text { major whole } \\
\text { tone, }=\mathbf{T}_{1}\end{array}$ & $\begin{array}{l}1 / \lambda= \\
(6,-9,-1)\end{array}$ \\
\hline $\begin{array}{l}\lambda^{2} \mu^{2} \rho= \\
(4,-8,1) \\
\text { grave minor } \\
\text { second }\end{array}$ & $\begin{array}{l}\lambda^{3} \mu^{3} \rho= \\
(9,-14,-1)\end{array}$ & $\begin{array}{l}\lambda^{4} \mu^{3} \rho= \\
(3,-5,0) \\
\text { limma, } \\
=\mathbf{s}_{3}=\boldsymbol{\alpha}\end{array}$ & $\begin{array}{l}\lambda^{5} \mu^{4} \rho= \\
(8,-11,-2)\end{array}$ & $\begin{array}{l}\lambda^{7} \mu^{5} \rho= \\
(7,-8,-3)\end{array}$ & $\begin{array}{l}\lambda^{8} \mu^{6} \rho^{2}= \\
(6,-10,0) \\
\text { Pythag. } \\
\text { diminished third }\end{array}$ & $\begin{array}{l}\lambda^{10} \mu^{7} \rho^{2}= \\
(5,-7,-1)\end{array}$ & $\begin{array}{l}\lambda / \mu= \\
(-17,24,4)\end{array}$ \\
\hline $\begin{array}{l}\lambda^{3} \mu \rho= \\
(-13,16,5)\end{array}$ & $\begin{array}{l}\lambda^{4} \mu^{2} \rho= \\
(-8,10,3)\end{array}$ & $\begin{array}{l}\lambda^{5} \mu^{3} \rho= \\
(-14,19,4)\end{array}$ & $\begin{array}{l}\lambda^{6} \mu^{3} \rho= \\
(-9,13,2)\end{array}$ & $\begin{array}{l}\lambda^{8} \mu^{4} \rho= \\
(-10,16,1)\end{array}$ & $\begin{array}{l}\lambda^{9} \mu^{5} \rho^{2}= \\
(-11,14,4)\end{array}$ & $\begin{array}{l}\lambda^{11} \mu^{6} \rho^{2}= \\
(-12,17,3)\end{array}$ & $\begin{array}{l}1 / \lambda= \\
(6,-9,-1)\end{array}$ \\
\hline $\begin{array}{l}\lambda^{2} \mu \rho= \\
(-7,7,4)\end{array}$ & $\begin{array}{l}\lambda^{3} \mu^{2} \rho \\
=(-2,1,2) \\
\text { minor chroma, } \\
=\mathbf{S}_{3}=\gamma\end{array}$ & $\begin{array}{l}\lambda^{4} \mu^{2} \rho= \\
(-8,10,3)\end{array}$ & $\begin{array}{l}\lambda^{5} \mu^{3} \rho \\
=(-3,4,1) \\
\text { major chroma, } \\
\text { major limma }\end{array}$ & \begin{tabular}{|l}
$\lambda^{7} \mu^{4} \rho=$ \\
$(-4,7,0)$ \\
apotome
\end{tabular} & $\begin{array}{l}\lambda^{8} \mu^{5} \rho^{2} \\
=(-5,5,3) \\
\text { double } \\
\text { augmented prime }\end{array}$ & $\begin{array}{l}\lambda^{10} \mu^{6} \rho^{2}= \\
(-6,8,2) \\
\text { (maj. chroma) }\end{array}$ & $\begin{array}{l}1 / \lambda= \\
(6,-9,-1)\end{array}$ \\
\hline $\begin{array}{l}\lambda \mu \rho= \\
(-1,-2,3) \\
\text { maximal } \\
\text { diesis }\end{array}$ & $\begin{array}{l}\lambda^{2} \mu^{2} \rho= \\
(4,-8,1) \\
\text { grave minor } \\
\text { second }\end{array}$ & $\begin{array}{l}\lambda^{3} \mu^{2} \rho= \\
(-2,1,2) \\
\text { minor chroma, } \\
=\mathbf{S}_{3}=\gamma\end{array}$ & $\begin{array}{l}\lambda^{4} \mu^{3} \rho= \\
(3,-5,0) \\
\operatorname{limma} \\
=\mathbf{s}_{3}=\boldsymbol{\alpha}\end{array}$ & $\begin{array}{l}\lambda^{6} \mu^{4} \rho= \\
(2,-2,-1) \\
\text { minor diatonic } \\
\text { semitone, }=\mathbf{S}\end{array}$ & $\begin{array}{l}\lambda^{7} \mu^{5} \rho^{2}= \\
(1,-4,2) \\
\text { grave } \\
\text { whole tone }\end{array}$ & $\begin{array}{l}\lambda^{9} \mu^{6} \rho^{2}= \\
(0,-1,1) \\
\text { minor whole } \\
\text { tone, }=\mathbf{T}_{2}\end{array}$ & $\begin{array}{l}1 / \lambda= \\
(6,-9,-1)\end{array}$ \\
\hline$\stackrel{\mu \rho}{=}(5,-11,2)$ & $\begin{array}{l}\lambda \mu^{2} \rho= \\
(10,-17,0) \\
\text { Pythag. double } \\
\text { dim. third }\end{array}$ & $\begin{array}{l}\lambda^{2} \mu^{2} \rho= \\
(4,-8,1) \\
\text { grave minor } \\
\text { second }\end{array}$ & $\begin{array}{l}\lambda^{3} \mu^{3} \rho= \\
(9,-14,-1)\end{array}$ & $\begin{array}{l}\lambda^{5} \mu^{4} \rho= \\
(8,-11,-2)\end{array}$ & $\begin{array}{l}\lambda^{6} \mu^{5} \rho^{2}= \\
(7,-13,1)\end{array}$ & $\begin{array}{l}\lambda^{8} \mu^{6} \rho^{2}= \\
(6,-10,0)\end{array}$ & $\begin{array}{l}\lambda^{3} \mu / \rho= \\
(-1,8,-5)\end{array}$ \\
\hline $\begin{array}{l}\lambda^{3} \mu^{2}= \\
(4-3,-3) \\
\text { minor diesis, } \\
=\mathbf{S}_{1}^{-1} \mathbf{p}^{-1}\end{array}$ & $\begin{array}{l}\lambda^{4} \mu^{3}= \\
(9,-9,-5)\end{array}$ & $\begin{array}{l}\lambda^{5} \mu^{3}=(3,0,-4) \\
\text { major diesis, } \\
=S_{1}^{-1}\end{array}$ & $\begin{array}{l}\lambda^{6} \mu^{4}= \\
(8,-6,-6) \\
(\text { min. diesis })^{2}\end{array}$ & $\begin{array}{l}\lambda^{8} \mu^{5}= \\
(7,-3,-7)\end{array}$ & $\begin{array}{l}\lambda^{9} \mu^{6} \rho= \\
(6,-5,-4) \\
\text { double dim. third }\end{array}$ & $\begin{array}{l}\lambda^{11} \mu^{7} \rho= \\
(5,-2,-5)\end{array}$ & $\begin{array}{l}\rho / \lambda^{3} \mu^{2}= \\
(-10,7,8)\end{array}$ \\
\hline $\begin{array}{l}\rho=(-6,4,5) \\
\text { small diesis }\end{array}$ & $\begin{array}{l}\lambda \mu \rho= \\
(-1,-2,3) \\
\text { maximal } \\
\text { diesis }\end{array}$ & $\begin{array}{l}\lambda^{2} \mu \rho= \\
(-7,7,4)\end{array}$ & $\begin{array}{l}\lambda^{3} \mu^{2} \rho=(-2,1,2) \\
\text { minor chroma, } \\
=\mathbf{S}_{3}=\gamma\end{array}$ & $\begin{array}{l}\lambda^{5} \mu^{3} \rho= \\
(-3,4,1) \\
\text { major chroma, } \\
\text { major limma }\end{array}$ & $\begin{array}{l}\lambda^{6} \mu^{4} \rho^{2}= \\
(-4,2,4) \\
\text { BP great } \\
\text { semitone }\end{array}$ & $\begin{array}{l}\lambda^{8} \mu^{5} \rho^{2} \\
=(-5,5,3) \\
\text { double } \\
\text { augmented prime }\end{array}$ & $\begin{array}{l}1 / \rho= \\
(6,-4,-5)\end{array}$ \\
\hline \multirow[t]{2}{*}{$\begin{array}{l}\mathbf{1}=(0,0,0) \\
\text { unison }\end{array}$} & $\begin{array}{l}\lambda \mu= \\
(5,-6,-2) \\
\text { diaschisma }\end{array}$ & $\begin{array}{l}\lambda^{2} \mu= \\
(-1,3,-1) \\
\text { syntonic } \\
\text { comma, }=\mathbf{p}\end{array}$ & $\begin{array}{l}\lambda^{3} \mu^{2}= \\
(4,-3,-3) \\
\text { minor diesis, } \\
=\mathbf{S}_{1}^{-1} \mathbf{p}^{-1}\end{array}$ & $\mid \begin{array}{l}\lambda^{5} \mu^{3}= \\
(3,0,-4) \\
\text { major diesis, } \mathbf{S}_{1}^{-1}\end{array}$ & $\begin{array}{l}\lambda^{6} \mu^{4} \rho= \\
(2,-2,-1) \\
\text { minor diatonic } \\
\text { semitone, }=\mathbf{S}\end{array}$ & $\begin{array}{l}\lambda^{8} \mu^{5} \rho= \\
(1,1,-2) \text { large } \\
\text { limma, BP sm. } \\
\text { semitone, } \mathbf{S}_{2}\end{array}$ & $\begin{array}{l}1 / \lambda \mu= \\
(-5,6,2)\end{array}$ \\
\hline & $\begin{array}{l}\mathbf{1}=(0,0,0) \\
\text { unison }\end{array}$ & $\begin{array}{l}\lambda=(-6,9,1) \\
\text { schisma }\end{array}$ & $\begin{array}{l}\lambda^{2} \mu=(-1,3,-1) \\
\text { syntonic } \\
\text { comma, }=\mathbf{p}\end{array}$ & \begin{tabular}{|l}
$\lambda^{4} \mu^{2}=(-2,6,-2)$ \\
Mathieu \\
superdiesis
\end{tabular} & $\begin{array}{l}\lambda^{5} \mu \rho=(-3,4,1) \\
\text { major chroma, } \\
\text { major limma }\end{array}$ & $\begin{array}{l}\lambda^{7} \mu^{4} \rho= \\
(-4,7,0) \\
\text { apotome }\end{array}$ & $\begin{array}{l}1 / \lambda= \\
(6,-9,-1)\end{array}$ \\
\hline \multirow[t]{5}{*}{$\begin{array}{l}\lambda^{5} \mu^{3} \rho=(-3,4,1) \\
\text { major chroma, } \\
\text { major limma }\end{array}$} & & $\begin{array}{l}\mathbf{1}=(0,0,0) \\
\text { unison }\end{array}$ & $\begin{array}{l}\lambda \mu=(5,-6,-2) \\
\text { diaschisma }\end{array}$ & \begin{tabular}{|l}
$\lambda^{3} \mu^{2}=(4,-3,-3)$ \\
minor diesis, \\
$=\mathbf{S}_{1}^{-1} \mathbf{p}^{-1}$
\end{tabular} & $\begin{array}{l}\lambda^{4} \mu^{3} \rho= \\
(3,-5,0) \text { limma, } \\
=\mathbf{s}_{3}=\boldsymbol{\alpha}\end{array}$ & $\begin{array}{l}\lambda^{6} \mu^{4} \rho= \\
(2,-2,-1) \\
\text { minor diatonic } \\
\text { semitone, }=\mathbf{S}\end{array}$ & $\begin{array}{l}1 / \lambda \mu= \\
(-5,6,2)\end{array}$ \\
\hline & $\begin{array}{l}\lambda^{6} \mu^{4} \rho= \\
(2,-2,-1) \\
\text { minor diatonic } \\
\text { semitone, }=\mathbf{S}\end{array}$ & & $\begin{array}{l}\mathbf{1}=(0,0,0) \\
\text { unison }\end{array}$ & $\begin{array}{l}\lambda^{2} \mu=(-1,3,-1) \\
\text { syntonic } \\
\text { comma, }=\mathbf{p}\end{array}$ & $\begin{array}{l}\lambda^{3} \mu^{2} \rho=(-2,1,2) \\
\text { minor chroma, } \\
=\mathbf{S}_{3}=\boldsymbol{\gamma}\end{array}$ & $\begin{array}{l}\lambda^{5} \mu^{3} \rho=(-3,4,1) \\
\text { major chroma, } \\
\text { major limma }\end{array}$ & $\begin{array}{l}1 / \lambda^{2} \mu= \\
(1,-3,1)\end{array}$ \\
\hline & & $\begin{array}{l}\lambda^{8} \mu^{5} \rho=(1,1,-2) \\
\text { large limma, BP } \\
\text { sm. semitone, } \mathbf{S}_{2}\end{array}$ & & $\begin{array}{l}\mathbf{1}=(0,0,0) \\
\text { unison }\end{array}$ & $\begin{array}{l}\lambda \mu \rho= \\
(-1,-2,3) \\
\text { maximal diesis }\end{array}$ & $\begin{array}{l}\lambda^{3} \mu^{2} \rho=(-2,1,2) \\
\text { minor chroma, } \\
=\mathbf{S}_{3}=\gamma\end{array}$ & $\begin{array}{l}1 / \lambda \mu \rho= \\
(1,2,-3)\end{array}$ \\
\hline & & & $\begin{array}{l}\lambda^{9} \mu^{6} \rho^{2}=(0,-1,1) \\
\text { minor whole } \\
\text { tone, }=\mathbf{T}_{2}\end{array}$ & & $\begin{array}{l}\mathbf{1}=(0,0,0) \\
\text { unison }\end{array}$ & $\begin{array}{l}\lambda^{2} \mu=(-1,3,-1) \\
\text { syntonic } \\
\text { comma, }=\mathbf{p}\end{array}$ & $\begin{array}{l}1 / \lambda^{2} \mu= \\
(1,-3,1)\end{array}$ \\
\hline & & & & $\begin{array}{l}\lambda^{11} \mu^{7} \rho^{2}=(-1,2,0) \\
\text { major whole } \\
\text { tone, }=\mathbf{T}_{1}\end{array}$ & & $\begin{array}{l}\mathbf{1}=(0,0,0) \\
\text { unison }\end{array}$ & \\
\hline
\end{tabular}


Table 4. List of 55 Intervals of Multiplication Table 3

The second column of this table lists the intervals in algebraic form best suited to exhibit the relationships between the intervals. The third column lists the intervals as lattice points of the lattice with basis $\lambda, \mu, \rho$. The fifth column lists the intervals as lattice points in the lattice with basis $(2 / 1),(3 / 2),(5 / 3)$ from which the quotients, given in the second to last column, are easily obtained. The numeral following a semicolon indicates the power of a factor 10 which cancels in the particular quotient. Finally, the last column lists the name of an interval as given in standard musical terminology. If no name for an interval is listed in this column then no standard name has been assigned to this interval. These intervals can be expressed in various forms in terms of the other intervals as is easily seen from the algebraic properties listed in column 2 . The symbols $S, s, T, \alpha, \gamma, p$ refer to musical systems discussed in ref. [1]

\begin{tabular}{|c|c|c|c|c|c|c|}
\hline $\begin{array}{l}\# \text { of } \\
\text { int. }\end{array}$ & $\begin{array}{l}\lambda \mu \rho \text { as } \\
\text { factors }\end{array}$ & $\begin{array}{l}\lambda \mu \rho \text { basis } \\
\text { as vectors }\end{array}$ & $\begin{array}{l}\text { Numerical } \\
\text { value of } \nu^{\prime} / \nu\end{array}$ & $\begin{array}{l}(2 / 1)(3 / 2)(5 / 3) \\
\text { basis }\end{array}$ & $\begin{array}{l}\text { Frequency } \\
\text { ratio } \nu^{\prime} / \nu\end{array}$ & $\begin{array}{l}\text { Name of } \\
\text { interval }\end{array}$ \\
\hline \#1 & 1 & {$[0,0,0]$} & 1.00000000 & $(0,0,0)$ & $1 / 1$ & unison \\
\hline$\# 2$ & $\lambda$ & {$[1,0,0]$} & 1.00112915 & $(-6,9,1)$ & $32,805 / 32,768$ & schisma \\
\hline$\# 3$ & $\lambda^{2}$ & {$[2,0,0]$} & 1.00225958 & $(-12,18,2)$ & $1.076168 / 1.073742 ; 9$ & $(\text { schisma })^{2}$ \\
\hline$\# 4$ & $\lambda^{3}$ & {$[3,0,0]$} & 1.00339128 & $(-18,27,3)$ & $3.530369 / 3.518437 ; 13$ & $(\text { schisma })^{3}$ \\
\hline$\# 5$ & $\rho / \lambda^{3} \mu$ & {$[-3,-1,1]$} & 1.00356076 & $(1,-8,5)$ & $1.600000 / 1.594323 ; 6$ & $\begin{array}{l}\text { Kleisma- } \\
\text { schisma }\end{array}$ \\
\hline$\# 6$ & $\rho / \lambda^{2} \mu$ & {$[-2,-1,1]$} & 1.00469393 & $(-5,1,6)$ & $15,625 / 15,552$ & Kleisma \\
\hline$\# 7$ & $\rho / \lambda \mu$ & {$[-1,-1,1]$} & 1.00582838 & $(-11,10,7)$ & $2.109375 / 2.097152 ; 6$ & $\begin{array}{l}\text { Fokker's } \\
\text { comma, } \\
\text { semicomma }\end{array}$ \\
\hline$\# 8$ & $\lambda^{3} \mu^{2} / \rho$ & {$[3,2,-1]$} & 1.00663296 & $(10,-7,-8)$ & $393,216 / 390,625$ & $\begin{array}{l}\text { Wuerschmidt's } \\
\text { comma }\end{array}$ \\
\hline$\# 9$ & $\rho / \mu$ & {$[0,-1,1]$} & 1.00696411 & $(-17,19,8)$ & $6.919805 / 6.871948 ; 10$ & \\
\hline$\# 10$ & $\mu / \lambda$ & {$[-1,1,0]$} & 1.00907794 & $(17,-24,-4)$ & $2.199023 / 2.179240 ; 12$ & \\
\hline \#11 & $\mu$ & {$[0,1,0]$} & 1.01021734 & $(11,-15,-3)$ & $6.710886 / 6.643012 ; 7$ & $\begin{array}{l}\text { diaschisma- } \\
\text { schisma }\end{array}$ \\
\hline$\# 12$ & $\lambda \mu$ & {$[1,1,0]$} & 1.01135802 & $(5,-6,-2)$ & $2,048 / 2,025$ & diaschisma \\
\hline \#13 & $\lambda^{2} \mu$ & {$[2,1,0]$} & 1.01250000 & $(-1,3,-1)$ & $81 / 80$ & $\begin{array}{l}\text { syntonic } \\
\text { comma, } \\
\text { Pythagorean } \\
\text { vector } p, \\
\text { Didymic } \\
\text { comma }\end{array}$ \\
\hline \#14 & $\lambda^{3} \mu$ & {$[3,1,0]$} & 1.01364326 & $(-7,12,0)$ & $531,441 / 524,288$ & $\begin{array}{l}\text { Pythagorean } \\
\text { comma, ditonic } \\
\text { comma, } s_{1}\end{array}$ \\
\hline
\end{tabular}


Table 4 (continued)

\begin{tabular}{|c|c|c|c|c|c|c|}
\hline $\begin{array}{l}\# \text { of } \\
\text { int. }\end{array}$ & $\begin{array}{l}\lambda \mu \rho \text { as } \\
\text { factors }\end{array}$ & $\begin{array}{l}\lambda \mu \rho \text { basis } \\
\text { as vectors }\end{array}$ & $\begin{array}{l}\text { Numerical } \\
\text { value of } \nu^{\prime} / \nu\end{array}$ & $\begin{array}{l}(2 / 1)(3 / 2)(5 / 3) \\
\text { basis }\end{array}$ & $\begin{array}{l}\text { Frequency } \\
\text { ratio } \nu^{\prime} / \nu\end{array}$ & $\begin{array}{l}\text { Name of } \\
\text { interval }\end{array}$ \\
\hline$\# 15$ & $\rho / \lambda$ & {$[-1,0,1]$} & 1.01610527 & $(0,-5,4)$ & $20,000 / 19,683$ & minimal diesis \\
\hline$\# 16$ & $\rho$ & {$[0,0,1]$} & 1.01725260 & $(-6,4,5)$ & $3,125 / 3,072$ & small diesis \\
\hline$\# 17$ & $\lambda^{2} \mu^{2}$ & {$[2,2,0]$} & 1.02284505 & $(10,-12,-4)$ & $4.194304 / 4.100625 ; 6$ & $(\text { diaschisma })^{2}$ \\
\hline$\# 18$ & $\lambda^{3} \mu^{2}$ & {$[3,2,0]$} & 1.02400000 & $(4,-3,-3)$ & $128 / 125$ & $\begin{array}{l}\text { minor diesis, } \\
\text { diesis, } S_{1}^{-1} p^{-1}\end{array}$ \\
\hline$\# 19$ & $\lambda^{4} \mu^{2}$ & {$[4,2,0]$} & 1.02515625 & $(-2,6,-2)$ & $6,561 / 6,400$ & $\begin{array}{l}\text { Mathieu } \\
\text { superdiesis, } \\
\text { (syntonic } \\
\text { comma) })^{2}\end{array}$ \\
\hline$\# 20$ & $\mu \rho$ & {$[0,1,1]$} & 1.02764622 & $(5,-11,2)$ & $1,638,400 / 1,594,323$ & \\
\hline$\# 21$ & $\lambda \mu \rho$ & {$[1,1,1]$} & 1.02806588 & $(-1,-2,3)$ & $250 / 243$ & $\begin{array}{l}\text { maximal } \\
\text { diesis }\end{array}$ \\
\hline$\# 22$ & $\lambda^{2} \mu \rho$ & {$[2,1,1]$} & 1.02996826 & $(-7,7,4)$ & $16,875 / 16,384$ & \\
\hline$\# 23$ & $\lambda^{3} \mu \rho$ & {$[3,1,1]$} & 1.03113125 & $(-13,16,5)$ & $5.535844 / 5.368709 ; 8$ & \\
\hline$\# 24$ & $\lambda^{4} \mu^{3}$ & {$[4,3,0]$} & 1.03563062 & $(9,-9,-5)$ & $262,144 / 253,125$ & \\
\hline$\# 25$ & $\lambda^{5} \mu^{3}$ & {$[5,3,0]$} & 1.03680000 & $(3,0,-4)$ & $648 / 625$ & major diesis, $S_{1}^{-1}$ \\
\hline$\# 26$ & $\lambda \mu^{2} \rho$ & {$[1,2,1]$} & 1.03931825 & $(10,-17,0)$ & $1.342 \quad 177 / 1.291402 ; 8$ & $\begin{array}{l}\text { Pythagorean } \\
\text { double } \\
\text { diminished } \\
\text { third, } s_{2}\end{array}$ \\
\hline$\# 27$ & $\lambda^{2} \mu^{2} \rho$ & {$[2,2,1]$} & 1.04049179 & $(4,-8,1)$ & $20,480 / 19,683$ & $\begin{array}{l}\text { grave minor } \\
\text { second }\end{array}$ \\
\hline$\# 28$ & $\lambda^{3} \mu^{2} \rho$ & {$[3,2,1]$} & 1.04166667 & $(-2,1,2)$ & $25 / 24$ & $\begin{array}{l}\text { minor chroma, } \\
\text { classic chromatic } \\
\text { semitone, } S_{3}, \gamma\end{array}$ \\
\hline$\# 29$ & $\lambda^{4} \mu^{2} \rho$ & {$[4,2,1]$} & 1.04284286 & $(-8,10,3)$ & $273,375 / 262,144$ & \\
\hline$\# 30$ & $\lambda^{5} \mu^{2} \rho$ & {$[5,2,1]$} & 1.04402039 & $(-14,19,4)$ & 9.968 067/8.589 935 & \\
\hline$\# 31$ & $\lambda^{6} \mu^{4}$ & {$[6,4,0]$} & 1.04857600 & $(8,-6,-6)$ & $16,384 / 15,625$ & \\
\hline \#32 & $\lambda^{3} \mu^{3} \rho$ & {$[3,3,1]$} & 1.05230973 & $(9,-14,-1)$ & $8,388,608 / 7,971,615$ & \\
\hline$\# 33$ & $\lambda^{4} \mu^{3} \rho$ & {$[4,3,1]$} & 1.05349794 & $(3,-5,0)$ & $256 / 243$ & $\begin{array}{l}\text { limma, Pyth. } \\
\text { min. second, } \\
s_{3}, \alpha\end{array}$ \\
\hline$\# 34$ & $\lambda^{5} \mu^{3} \rho$ & {$[5,3,1]$} & 1.05468750 & $(-3,4,1)$ & $135 / 128$ & $\begin{array}{l}\text { major chroma } \\
\text { major limma }\end{array}$ \\
\hline
\end{tabular}


Table 4 (continued)

\begin{tabular}{|c|c|c|c|c|c|c|}
\hline $\begin{array}{l}\# \text { of } \\
\text { int. }\end{array}$ & $\begin{array}{l}\lambda \mu \rho \text { as } \\
\text { factors }\end{array}$ & $\begin{array}{l}\lambda \mu \rho \text { basis } \\
\text { as vectors }\end{array}$ & $\begin{array}{l}\text { Numerical } \\
\text { value of } \nu^{\prime} / \nu\end{array}$ & $\begin{array}{l}(2 / 1)(3 / 2)(5 / 3) \\
\text { basis }\end{array}$ & $\begin{array}{l}\text { Frequency } \\
\text { ratio } \nu^{\prime} / \nu\end{array}$ & $\begin{array}{l}\text { Name of } \\
\text { interval }\end{array}$ \\
\hline$\# 35$ & $\lambda^{6} \mu^{3} \rho$ & {$[6,3,1]$} & 1.05587840 & $(-9,13,2)$ & $4,428,675 / 4,194304$ & \\
\hline$\# 36$ & $\lambda^{8} \mu^{5}$ & {$[8,5,0]$} & 1.06168320 & $(7,-3,-7)$ & $82,944 / 78,125$ & \\
\hline$\# 37$ & $\lambda^{5} \mu^{4} \rho$ & {$[5,4,1]$} & 1.06546360 & $(8,-11,-2)$ & $524,288 / 492,075$ & \\
\hline \#38 & $\lambda^{6} \mu^{4} \rho$ & {$[6,4,1]$} & 1.06666667 & $(2,-2,-1)$ & $16 / 15$ & $\begin{array}{l}\text { minor diatonic } \\
\text { semitone, } S\end{array}$ \\
\hline \#39 & $\lambda^{7} \mu^{4} \rho$ & {$[7,4,1]$} & 1.06787109 & $(-4,7,0)$ & $2,187 / 2,048$ & apotome \\
\hline$\# 40$ & $\lambda^{8} \mu^{4} \rho$ & {$[8,4,1]$} & 1.06907688 & $(-10,16,1)$ & $7.174553 / 6.710886 ; 7$ & \\
\hline$\# 41$ & $\lambda^{7} \mu^{5} \rho$ & {$[7,5,1]$} & 1.07878189 & $(7,-8,-3)$ & $32,768 / 30,375$ & \\
\hline$\# 42$ & $\lambda^{8} \mu^{5} \rho$ & {$[8,5,1]$} & 1.08000000 & $(1,1,-2)$ & $27 / 25$ & $\begin{array}{l}\text { large limma, } \\
\text { BP small } \\
\text { semitone }\end{array}$ \\
\hline$\# 43$ & $\lambda^{6} \mu^{4} \rho^{2}$ & {$[6,4,2]$} & 1.08506944 & $(-4,2,4)$ & $625 / 576$ & $\begin{array}{l}\text { BP great } \\
\text { semitone }\end{array}$ \\
\hline$\# 44$ & $\lambda^{9} \mu^{6} \rho$ & {$[9,6,1]$} & 1.09226667 & $(6,-5,-4)$ & $2,048 / 1,875$ & $\begin{array}{l}\text { double } \\
\text { diminished } \\
\text { third }\end{array}$ \\
\hline$\# 45$ & $\lambda^{6} \mu^{5} \rho^{2}$ & {$[6,5,2]$} & 1.09615597 & $(7,-13,1)$ & $5.24288 / 4.782969 ; 6$ & \\
\hline$\# 46$ & $\lambda^{7} \mu^{5} \rho^{2}$ & {$[7,5,2]$} & 1.09739369 & $(1,-4,2)$ & $800 / 729$ & $\begin{array}{l}\text { grave whole } \\
\text { tone }\end{array}$ \\
\hline$\# 47$ & $\lambda^{8} \mu^{5} \rho^{2}$ & {$[8,5,2]$} & 1.09863281 & $(-5,5,3)$ & $1,125 / 1,024$ & $\begin{array}{l}\text { double } \\
\text { augmented } \\
\text { prime }\end{array}$ \\
\hline$\# 48$ & $\lambda^{9} \mu^{5} \rho^{2}$ & {$[9,5,2]$} & 1.09987333 & $(-11,14,4)$ & $3.690562 / 3.355443 ; 7$ & \\
\hline$\# 49$ & $\lambda^{11} \mu^{7} \rho$ & {$[11,7,1]$} & 1.10592000 & $(5,-2,-5)$ & $3,456 / 3,125$ & \\
\hline$\# 50$ & $\lambda^{8} \mu^{6} \rho^{2}$ & {$[8,6,2]$} & 1.10985791 & $(6,-10,0)$ & $65,536 / 59,049$ & $\begin{array}{l}\text { Pyth. } \\
\text { diminished } \\
\text { third }\end{array}$ \\
\hline$\# 51$ & $\lambda^{9} \mu^{6} \rho^{2}$ & {$[9,6,2]$} & 1.11111111 & $(0,-1,1)$ & $10 / 9$ & $\begin{array}{l}\text { minor whole } \\
\text { tone } T_{2}\end{array}$ \\
\hline$\# 52$ & $\lambda^{10} \mu^{6} \rho^{2}$ & {$[10,6,2]$} & 1.11236572 & $(-6,8,2)$ & $18,225 / 16,384$ & \\
\hline$\# 53$ & $\lambda^{11} \mu^{6} \rho^{2}$ & {$[11,6,2]$} & 1.11362175 & $(-12,17,3)$ & 8.978 711/5.368 709 & \\
\hline$\# 54$ & $\lambda^{10} \mu^{7} \rho^{2}$ & {$[10,7,2]$} & 1.12373114 & $(5,-7,-1)$ & $4,096 / 3,645$ & \\
\hline \#55 & $\lambda^{11} \mu^{7} \rho^{2}$ & {$[11,7,2]$} & 1.12500000 & $(-1,2,0)$ & $9 / 8$ & $\begin{array}{l}\text { major whole } \\
\text { tone } T_{1}\end{array}$ \\
\hline
\end{tabular}


A few examples will illustrate the properties among tones as given by the table:

$$
\begin{aligned}
& \mathbf{t}=[n, m, r]=\lambda^{n} \mu^{m} \rho^{r}, \quad n, m, r \text { integers },
\end{aligned}
$$

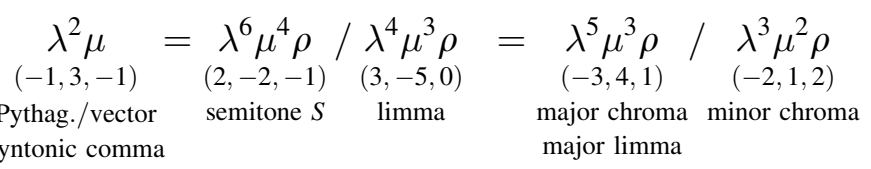

$$
\begin{aligned}
& =\underset{(1,1,-2)}{\lambda^{8} \mu^{5} \rho} / \underset{(2,-2,-1)}{\lambda^{6} \mu^{4} \rho}=\underset{(-1,2,0)}{\lambda^{11} \mu^{7} \rho^{2}} / \begin{array}{c}
\lambda^{9} \mu^{6} \rho^{2} \\
(0,-1,1)
\end{array} \\
& \text { large limma minor diatonic major whole tone minor whole tone } \\
& \text { BP small semitone semitone }
\end{aligned}
$$

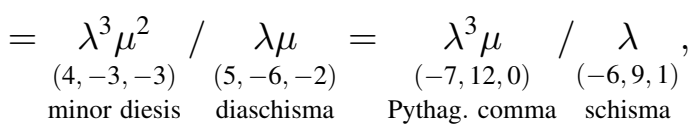

$\mu($ diaschisma-schisma $)=(\lambda \mu)^{2}(\text { diaschisma })^{2} /\left(\lambda^{2} \mu\right)$ syntonic comma

$$
=\left(\lambda^{3} \mu^{2}\right) \text { diesis minor } /\left(\lambda^{3} \mu\right) \text { Pythagorean comma, }
$$

$\rho / \mu=\left(\lambda^{5} \mu^{3} \rho\right)$ major chroma $/\left(\lambda^{3} \mu^{2}\right)$ minor diesis $\cdot(\lambda \mu)$ diaschisma $\cdot(\lambda \mu \rho)$ maximal diesis

$=\left(\lambda^{3} \mu\right)$ Pythagorean comma $/\left(\lambda^{3} \mu \rho\right)$ Wuerschmidt's comma $=(\rho)$ small diesis

$\cdot\left(\lambda^{3} \mu\right)$ Pythagorean comma $/\left(\lambda^{3} \mu^{2}\right)$ diesis minor,

$\lambda($ schisma $)=\left(\lambda^{3} \mu^{2} \rho\right)$ minor chroma $/(\lambda \mu \rho)$ maximal diesis $\cdot(\lambda \mu)$ diaschisma

$$
=(\rho / \lambda \mu) \text { Fokker's comma } /\left(\rho / \lambda^{2} \mu\right) / \text { Kleisma, }
$$

etc.

Given any two tones $\mathbf{t}_{3}, \mathbf{t}_{3}^{\prime}$, these tones can be connected by means of a consecutive sequence of adjacent factors $f \rightarrow, f \downarrow$, leading to a factorization of tones,

$$
\mathbf{t}_{3}^{\prime} \cdot f \rightarrow \cdot f \rightarrow \cdot f \downarrow \cdot f \rightarrow \cdots=\mathbf{t}_{3} .
$$


For example,

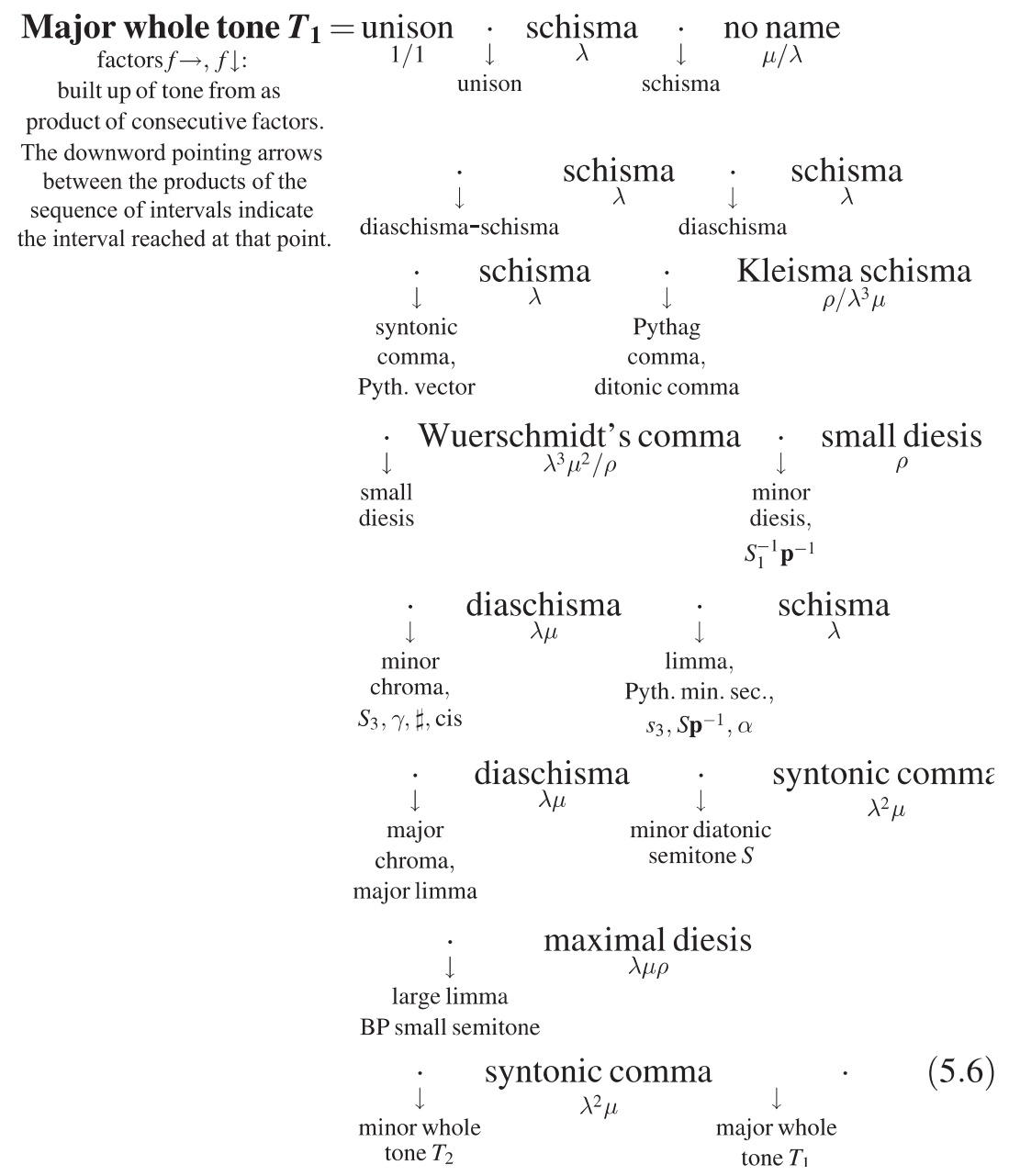

\section{The Octave Multiplication Table}

The Multiplication Table 3 is limited to the interval $[c, d]$. It will be shown in this section that this table can be used, with minor modification, for the tones/intervals of the entire octave. This is achieved by using again symmetry considerations, namely translations in the musical lattice space.

Table 5 illustrates the procedure. In this table the small triangle in the uppermost left corner represents Table 3. This triangle, 
Table 5. Multiplication-Division Table for Full Octave. This table represents the reduced multiplication table for the 116 tones/intervals of the octave. (The full table would contain $116 \times 116$ entries.) Note that the tones/intervals of $T_{2}$ and $S$ form subsets of the set of tones of $T_{1}$. $T_{1}=$ major whole tone, $T_{2}=$ minor whole tone, $S=$ minor diatonic semitone, $(n, m, r)=(2 / 1)^{n}(3 / 2)^{m}(5 / 3)^{r}=$ $\left(c^{1}\right)^{n}(g)^{m}\left(c^{1}\right)^{r}, c^{1}=2 / 1, g=3 / 2, a=5 / 3$

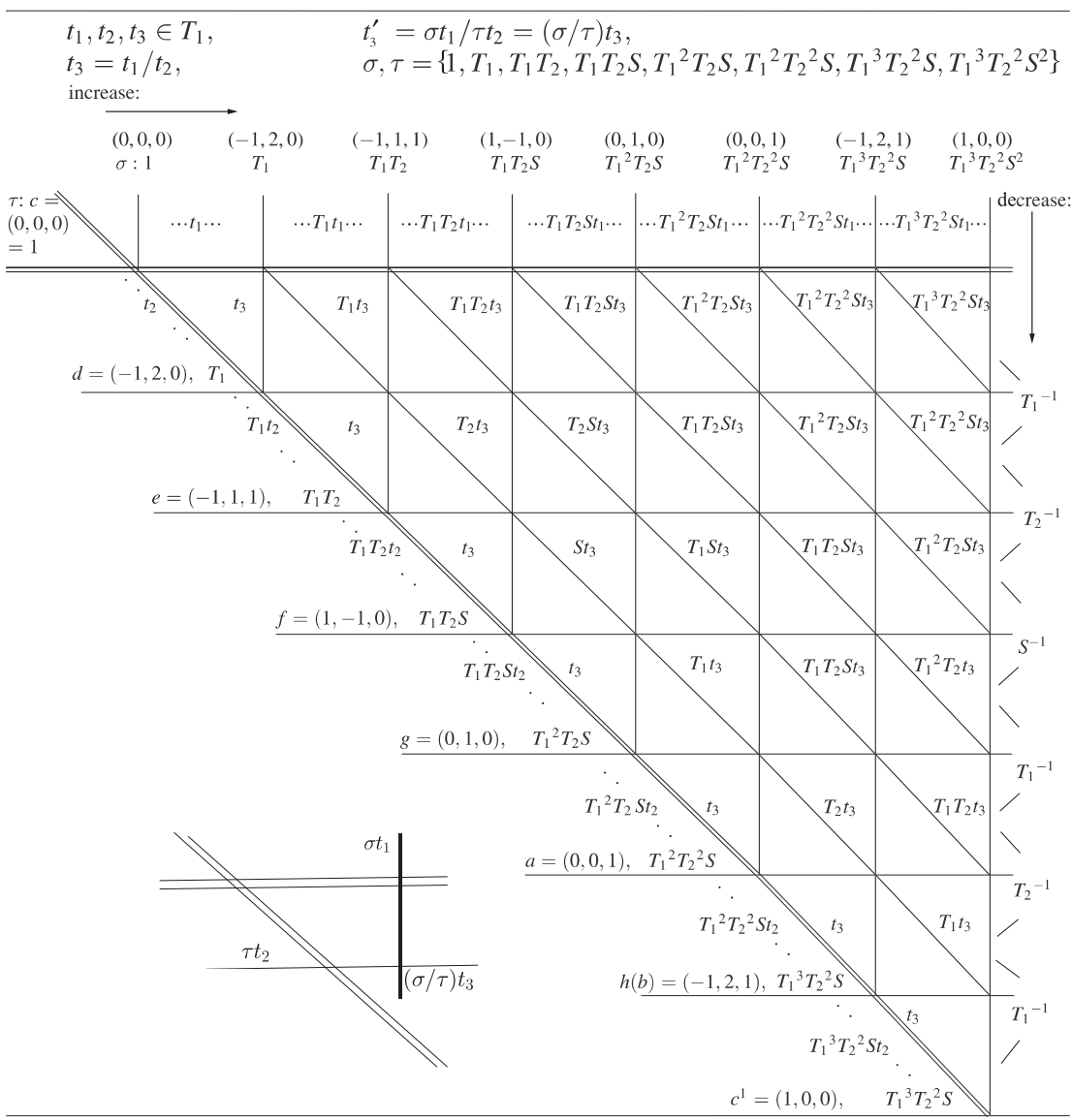

corresponding to the reduced Multiplication Table 3 for the interval $[c, d]$, can be translated to any point in the musical lattice. These translations are indicated by the other triangles of the diagram.

Such a shift (translation) of the basic trangle, namely Table 3 , is achieved by means of a constant multiplicative factor (or equivalently, using the vector notation for the musical tones, by means 
of an addition of a constant vector to the tonal vectors of Table 3 ). That is

$$
\mathbf{t}_{1} \rightarrow \sigma \mathbf{t}_{1}, \quad \mathbf{t}_{2} \rightarrow \tau \mathbf{t}_{2}, \quad \mathbf{t}_{3} \rightarrow(\sigma / \tau) \mathbf{t}_{3}
$$

with

$$
\begin{aligned}
\sigma, \tau= & \in\left\{1, T_{1}, T_{2}, T_{1} T_{2}, T_{1} T_{2} S, T_{1}^{2} T_{2} S, T_{1}^{2} T_{2}^{2} S, T_{1}^{3} T_{2}^{2} S\right\} \\
=\{ & (0,0,0)=c,(-1,2,0)=d,(-1,1,1)=e,(1,-1,0)=f, \\
& (0,1,0)=g,(0,0,1)=a,(-1,2,1)=h(b)\} \\
& T_{1}=(-1,2,0)=[11,7,2], \quad \text { major whole tone }, \\
& T_{2}=(0,-1,1)=[9,6,2], \quad \text { minor whole tone }, \\
& S=(2,-2,-1)=[6,4,1], \quad \text { semitone. }
\end{aligned}
$$

The interval $T_{1}$ contains more subintervals than the intervals $T_{2}$ and $S$. Thus $T_{1} \cdot \mathbf{t}_{1}, \mathbf{t}_{2} \in T_{1}$, will produce some tones which belong to the next adjacent interval. These outside lying tones need to be ignored if attention is focused on the tones of a specific interval only.

Given any two tones/intervals $\mathbf{t}_{1}^{\prime}, \mathbf{t}_{2}^{\prime}$, of the octave $\left[c, c^{1}\right]$ these two tones can be mapped upon two tones $\mathbf{t}_{1}, \mathbf{t}_{2}$, of the interval $[c, d]$, the major whole tone $T_{1}$,

$$
\mathbf{t}_{1}^{\prime}=\sigma \mathbf{t}_{1}, \quad \mathbf{t}_{2}^{\prime}=\tau \mathbf{t}_{2} .
$$

The (basic) Multiplication Table 3 for the interval $[c, d]$ however yields

$$
\mathbf{t}_{3}=\mathbf{t}_{1} / \mathbf{t}_{2}
$$

and thus

$$
\mathbf{t}_{3}^{\prime}=\mathbf{t}_{1}^{\prime} / \mathbf{t}_{2}^{\prime}=(\sigma / \tau)\left(\mathbf{t}_{1} / \mathbf{t}_{2}\right)=(\sigma / \tau) \mathbf{t}_{3} .
$$

Thus the product/division of any pair of tones $\mathbf{t}_{1}^{\prime}, \mathbf{t}_{2}^{\prime}$ from within any given interval, or from within two distinct intervals, can be reduced to a product of tones $\mathbf{t}_{1}, \mathbf{t}_{2}$ of the basic interval, Table 3 (and ultimately to the property of the tones $\lambda, \mu, \rho$ of the natural basis, Table 1).

An example will illustrate this property: Choosing

$$
\mathbf{t}_{1}^{\prime}=(4,-4,-1) \in\left[h(b), c^{1}\right], \quad \sigma=h(b)=T_{1}^{3} T_{2}^{2} S=(-1,2,1),
$$


with $\mathbf{t}_{1}^{\prime}$ the diminished octave. The tone/subinterval $\mathbf{t}_{1} \in[c, d]$ corresponding to the tone $\mathbf{t}_{1}^{\prime}$ is then given as

$$
\mathbf{t}_{1}=\mathbf{t}_{1}^{\prime} / \sigma=(4,-4,-1)-(-1,2,-1)=(5,6,-2) \in[c, d],
$$

the diaschisma. For $\mathbf{t}_{2}^{\prime}$ the tone/interval is chosen as

$$
\mathbf{t}_{2}^{\prime}=(-1,0,2) \in[f, g], \quad \tau=f=T_{1} T_{2} S=(1,-1,0),
$$

with $\mathbf{t}_{2}^{\prime}$ the minor augmented fourth. The corresponding tone/ interval $\mathbf{t}_{2} \in[c, d]$ is given as

$$
\mathbf{t}_{2}=\mathbf{t}_{2}^{\prime} / \tau=(-1,0,2)-(1,-1,0)=(-2,1,2) \in[c, d],
$$

with $\mathbf{t}_{2}$ the minor chroma. Then

$$
\mathbf{t}_{3}^{\prime}=(-2,3,1) \mathbf{t}_{3},
$$

with $(-2,3,1)$ the diatonic tritone. The tone $\mathbf{t}_{3}$, taken from Table 3 for $[c, d]$, is given as

$$
\begin{aligned}
\mathbf{t}_{3} & =\mathbf{t}_{1} / \mathbf{t}_{2}=(5,-6,-2)-(-2,1,2)=(7,-7,-4) \\
& =(1 /(-7,7,4)) .
\end{aligned}
$$

The ratio $\mathbf{t}_{3}^{\prime}$ of the tones $\mathbf{t}_{1}^{\prime}$ and $\mathbf{t}_{2}^{\prime}$ is given as

$$
\begin{aligned}
\mathbf{t}_{3}^{\prime}= & (-2,3,1)+(7,-7,-4)=(5,-4,-3), \\
& \text { the double diminished fifth. }
\end{aligned}
$$

Thus the following properties have been obtained:

diminished octave $(4,-4,-1)$ / classic augmented fourth $(-1,0,2)$

$=$ diatonic tritone $(-2,3,1)$

- $($ diashisma $(5,-6,-2) /$ minor chroma $(-2,1,2))$

$=$ double diminished fifth $(5,-4,-3)$,

major chroma $(-3,4,1)$ /minor diesis $(4,-3,-3)$

$=(-7,7,4)=$ minor chroma $(-2,1,2) /$ diashisma $(5,-6,-2)$.

\section{Acknowledgments}

The author wishes to thank Dieter Flury, Miguel Paramo Lorente, Peter

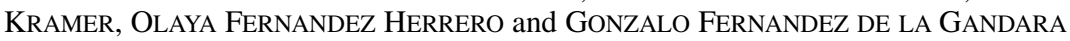
for their interest and comments. 
36 B. J. Gruber: Mathematical-Physical Properties of Musical Tone Systems III

\section{References}

[1] Gruber, B. J. (2005) Mathematical-Physical Properties of Musical Tone Systems. Sitzungsber. Öst. Akad. Wiss. Wien, math.-nat. Kl., Abt. II 214: 43-79

[2] Gruber, B. J. (2006) Mathematical-Physical Properties of Musical Tone Systems. II: Applications. Sitzungsber. Öst. Akad. Wiss. Wien, math.-nat. Kl., Abt. II 215: $45-105$

[3] Mazzola, G. (1990) Geometrie der Töne. Birkhäuser, Basel

[4] RiEmann, H. (1970) Dictionary of Music, pp. 796-801. Angerer \& Co, London; Da Capo Press, New York (Music Theory Reprint Series)

[5] HUYGENS-FOKKER, S.: List of Intervals [http://www.xs4all.nl/ huygensf/doc/ intervals.html]

Author's address: Prof. Bruno J. Gruber, Emeritus, College of Science, Southern Illinois University, Mailcode 4403, Carbondale, IL 62901, USA. E-Mail: Gruber@siu.edu. 